\title{
One-way quasiplanar terahertz absorbers using nonstructured polar dielectric layers
}

\author{
P. Rodríguez-Ulibarri, ${ }^{1}$ M. Beruete, ${ }^{1,2}$ and A. E. Serebryannikov ${ }^{3}$ \\ ${ }^{1}$ Antennas Group-TERALAB, Universidad Pública de Navarra, 31006 Pamplona, Spain \\ ${ }^{2}$ Institute of Smart Cities, Universidad Pública de Navarra, 31006 Pamplona, Spain \\ ${ }^{3}$ Faculty of Physics, Adam Mickiewicz University, 61-614 Poznań, Poland
}

(Received 17 June 2017; revised manuscript received 6 October 2017; published 30 October 2017)

\begin{abstract}
A concept of quasiplanar one-way transparent terahertz absorbers made of linear isotropic materials is presented. The resulting structure consists of a homogeneous absorbing layer of polar dielectric, GaAs, a dispersion-free substrate, and an ultrathin frequency-selective reflector. It is demonstrated that perfect absorption can be obtained for forward illumination, along with total reflection at backward illumination and transparency windows in the adjacent bands. The design is particularized for the polaritonic gap range where permittivity of GaAs varies in a wide range and includes epsilon-near-zero and transparency regimes. The underlying physics can be explained with the aid of a unified equivalent-circuit (EC) analytical model. Perfect matching of input impedance in forward operation and, simultaneously, strong mismatch in the backward case are the universal criteria of one-way absorption. It is shown that perfect one-way absorption can be achieved at rather arbitrary permittivity values, provided these criteria are fulfilled. The EC results are in good agreement with full-wave simulations in a wide range of material and geometrical parameters. The resulting one-way absorbers are very compact and geometrically simple, and enable transparency in the neighboring frequency ranges and, hence, multifunctionality that utilizes both absorption- and transmission-related regimes.
\end{abstract}

DOI: 10.1103/PhysRevB.96.155148

\section{INTRODUCTION}

The terahertz $(\mathrm{THz})$ frequency range is nowadays attracting more and more attention owing to its high potential for numerous day-to-day applications such as security, quality control processes, and biomedicine, to name a few [1]. The promising applications foreseen in these and other fields along with the discovery of efficient $\mathrm{THz}$ emitters and detectors have boosted both basic and applied research in the last decade.

Among the developed devices, $\mathrm{THz}$ absorbers have been extensively studied for imaging and sensing purposes. Classical microwave absorbers were initially based on $\lambda / 4$ lossy dielectric/magnetic slabs backed with metallic reflectors $[2,3]$. Later, within the realms of metamaterials, metasurfaces, and plasmonics, advanced absorber architectures have been demonstrated, enabling perfect and broadband absorption with ultrathin structures [4-12]. Typically, they are based on periodic arrays of subwavelength elements.

According to one of the recently suggested theoretical concepts [13], an arbitrary body can be perfectly absorbing if a precise amount of dissipation is added under some specific conditions. Coherent perfect absorption is considered as a very general phenomenon connected with the properties of the scattering matrix ( $\mathbb{S}$ matrix) and is mainly due to the common effect of interference and dissipation [13]. At the same time, a growing interest towards the realization of perfect absorption with homogeneous (nonstructured) layers has arisen in the last years. It has been demonstrated that perfect absorption can be obtained in reflection mode for an arbitrary thin layer, provided that the real parts of permittivity and permeability are zero even with arbitrarily small (but nonzero) imaginary parts [14]. Later, the theory of coherent perfect absorption in arbitrarily thin epsilon-near-zero (ENZ) layers was presented [15]. The ability of perfect absorption in these structures is often connected with the specific properties of the so-called ENZ modes [16]. Recently, perfect absorption has been shown by replacing a homogeneous ENZ layer by a dielectric grating with period $\lambda_{0} / 2<p<\lambda_{0}\left(\lambda_{0}\right.$ is free-space wavelength) made of conventional dielectrics [17]. In addition, perfect absorption can also be achieved in two-dimensional rod media with a small imaginary permittivity part [18], so it is not a unique property of thin homogeneous layers and isolated bodies. Likewise, dispersive natural materials showing a transition from plasmonic to dielectric state, thus enabling a wide range of permittivity variation and including ENZ and transparency regions, have been studied in the context of efficient absorption. For instance, it can be obtained with the aid of indium tin oxide at near infrared $[19,20]$ and GaAs at $\mathrm{THz}[18,21]$ frequencies. A part of the recent achievements in compact layered absorbers has been reviewed in Refs. [22,23].

From a multifunctionality perspective, physical scenarios and devices able to perform two functions in one step at fixed frequency or different functions in nearby frequency bands in one device are very promising [24-28]. An interesting realization from a practical viewpoint is to get perfect absorption in a given frequency band along with transparency in an adjacent band. For example, a metasurface able to work as a broadband microwave absorber, while being transparent at optical frequencies, has been proposed [27]. Transparency and absorption have been obtained in Ref. [21] in neighboring $\mathrm{THz}$ frequency bands, indicating a route to multifunctionality of the second type. This has been done in the framework of the concept of one-wayabsorbers that requires only isotropic, linear, and passive materials, and implemented using photonic crystals ( $\mathrm{PhCs}$ ) with broken spatial inversion symmetry, including those combining a $\mathrm{PhC}$ with a thin homogeneous absorbing layer. In particular, it has been shown that perfect absorption can be achieved at the upper edge of the polaritonic gap in the forward illumination case, whereas total reflection appears in the backward illumination case together with nearby two-way transparency bands. 
Among the wide variety of quasiplanar absorber designs, it is worth mentioning those showing asymmetric operation in which absorption occurs for illumination from one side only, see Ref. [23] and references therein. Most of them are based on scatterer arrays, but they can also be based on homogeneous layers. However, these absorbers do not ensure transparency in the neighboring bands in the same structure and thus cannot be used in multifunctional scenarios that require transparency windows. Moreover, they do not exploit the specific behavior of materials with strongly dispersive features. Very recently, structures with $\mathcal{P} T$ symmetry and, thus, with lossy and gain components have been suggested for unidirectional absorption [29]. However, gain components are not necessary [21,23].

In this paper, we develop the concept of quasiplanar transparent one-way $\mathrm{THz}$ absorbers and demonstrate it in structures comprising a thin homogeneous absorbing layer of polar dielectric (PD), a dispersion-free substrate layer, and a frequency-selective reflector (FSR). All the materials used are linear, passive, and isotropic. The main aims of this study are to clarify the conditions at which nearly perfect one-way absorption can appear in quasiplanar structures with a thin homogeneous absorbing layer, and justify the generality of the suggested concept and possibility of implementation of a universal model for different ranges of PD permittivity, together with the comparison of sample designs. A goal beyond the perfect one-way absorption is to test what is feasible from a multifunctional point of view. Therefore, we investigate whether one-way absorption is feasible simultaneously with other regimes in the frequency ranges which are more or less close to the one-way absorption band. To this end, a systematic study of the maximum absorption regimes under variation of the basic geometrical parameters is conducted by means of an equivalent-circuit (EC) model developed from the first-principles [30] and full-wave simulations from CST MICROWAVE STUDIO ${ }^{\circledR}$ (CST MWS), a commercial solver based on the finite integration method [31].

This study focuses on the polaritonic gap range and its vicinity, where fast variation in permittivity occurs for most PDs. It will be shown that the physical mechanism can be easily understood from the EC model, making it clear that perfect impedance match at forward-case illumination and strong mismatch at the backward-case illumination create the universal criteria for one-way absorption, regardless of the complex permittivity of the PD layer. The unambiguity of these criteria is indicated. With this methodology we demonstrate extremely compact one-way absorbers with total thickness less than $\lambda_{0} / 10$ in the high-absorption regime. An important feature of the suggested concept is the possibility of obtaining two-way transparency bands adjacent to the one-way absorption band that allows realization of multifunctionality within a rather narrow frequency range. This is typically not available in the recently proposed absorbers [5-11,23,27]. This unusual combination of transmission, reflection, and absorption properties at forward and backward illumination cases opens a way to realization of new functionalities useful for several $\mathrm{THz}$ applications. For instance, some $\mathrm{THz}$ generation techniques rely on nonlinear effects and may generate undesired harmonics. Our concept enables devices that may contribute to select a spectral window (transparency band) from the source while showing high absorption in an undesired frequency band. Another potential application of the presented device could be as part of a multifrequency reflector system where transmission/reception performance could be combined with separation of transmitter and receiver signals. In addition, it should be noted that this concept, in fact, is not limited to the $\mathrm{THz}$ regime. By using $\mathrm{SiC}$ as $\mathrm{PD}$ material, for instance, we can attain similar behavior within the mid-infrared (MIR) regime where thermal-related applications such as passive cooling [32] require multifunctionality performance.

\section{THEORETICAL BASICS}

There are various natural absorbing materials that show strong absorption at $\mathrm{THz}$ frequencies. In particular, PDs have both strong absorption and dispersion at $\mathrm{THz}$ frequencies due to the coupling of transverse phonon-photon resonances [33]. This gives rise to a frequency range with a negative real part of permittivity (polaritonic gap) and high losses. The natural behavior of these materials is connected to many interesting phenomena, even when homogeneous PD layers are used [21,34]. Their variety can be extended farther by introducing periodicity in one- and two-dimensional structures with PD components, which allows obtaining band splitting, asymmetric transmission, subwavelength guiding, hyperbolic dispersion, near-field resolution enhancement, and efficient control of absorption [34-40].

The Lorentz reciprocity in the studied case results in the following relations [41]:

$$
T^{\rightarrow}=T^{\leftarrow}, R^{\rightarrow} \neq R^{\leftarrow}, A^{\rightarrow} \neq A^{\leftarrow},
$$

where $T, R, A$ stand for transmittance, reflectance, and absorption, respectively, and $\rightarrow$ and $\leftarrow$ correspond to forward and backward illumination cases. Since for reciprocal structures like the ones treated here $T$ does not depend on the illumination direction (i.e., forward or backward), perfect absorption cannot be obtained without the condition of $T=0$. Then, energy conservation and reciprocity yield

$$
A^{\rightarrow}+R^{\rightarrow}=A^{\leftarrow}+R^{\leftarrow}
$$

In the ideal case of one-way absorption, $R^{\rightarrow}=0$ and $A^{\leftarrow}=0$, so the following conditions should be fulfilled:

$$
A^{\rightarrow}=1, \quad R^{\leftarrow}=1 .
$$

Figure 1(a) schematically depicts the one-way absorption scenario in a quasiplanar structure, in which the conditions (3) are fulfilled. The first equation in (3) invokes (i) impedance matching and, thus, zero reflection in steady state at the air-PD interface (see Ref. [42]) and (ii) total reflection at the substrate-FSR interface for the forward illumination case. Conversely, the second equation in (3) means that total reflection must be achieved at the air-FSR interface for the backward illumination case. In total, there are three conditions in terms of impedance of individual components and surrounding air that must be simultaneously satisfied.

These conditions can be fulfilled, for instance, by implementing a FSR made of periodically spaced rectangular patch elements patterned on a substrate layer with relative 


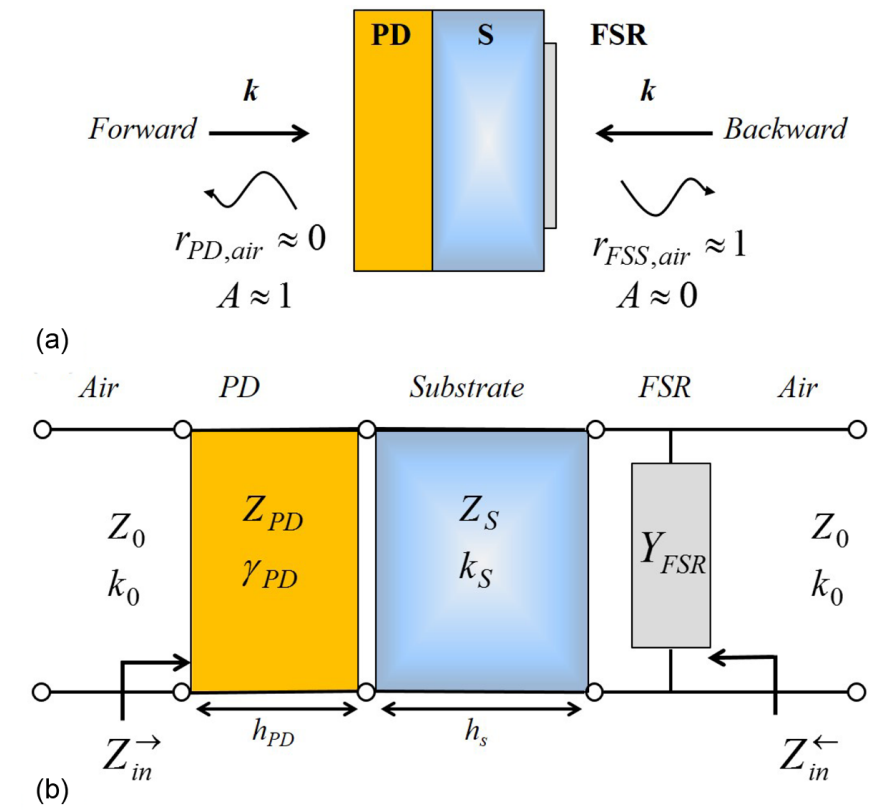

FIG. 1. (a) General geometry and operation basics of one-way absorber. The PD layer is attached to a nondispersive substrate (S) patterned with a FSR. (b) EC model of the studied structure: $Z_{0}, Z_{P D}$, and $Z_{S}$ are intrinsic impedances of free space, PD, and the substrate, respectively; $k_{0}, \gamma_{P D}$, and $k_{S}$ are free-space wave number, complex propagation constant of PD, and substrate wave number; $h_{P D}$ and $h_{S}$ stand for thicknesses of PD layer and substrate.

permittivity $\varepsilon_{S}=2.25$, which corresponds to polypropylene, a typical low-loss substrate for $\mathrm{THz}$ applications [43], see Fig. 1(a). As shown in the EC schematic in Fig. 1(b), the FSR can be modeled as an lumped-shunt admittance, $Y_{F S R}$, according to Ref. [30]. With this approach the array is considered to be infinite and periodic, and therefore the surface current of the patch can be expanded in terms of Floquet modes. This expansion leads to an equivalent circuit wherein the coupling coefficients of each harmonic are set as transformer turn ratios. The admittance $Y_{F S R}$ consists of the series connections of an inductance $L$ and a capacitance $C$ that account for the TE and TM high-order harmonics, respectively, and the distributed contribution of low-order harmonics which are either in propagation or evanescent but close to their cutoff. This approach is combined with classical transmission-line formulation [44], i.e., cascading the individual $T$ matrices of the PD layer, substrate, and FSR [see Fig. 1(b)]. In this model, the PD layer can be treated as a lossy and dispersive transmission line of length $h_{P D}$, with both complex characteristic impedance and propagation constant (see below) and the polypropylene layer as a lossless transmission line of length $h_{S}$ and propagation constant $\beta_{S}=$ $k_{0} \sqrt{\varepsilon_{S}}$. The reason for choosing the patch element as FSR is twofold. First, closed-form analytical equations are available, simplifying and speeding up the analysis and design process. Second, it is probably the simplest geometry that exhibits the reflection properties required for one-way absorption. It should be pointed out that for the orthogonal polarization (electric field parallel to the short patch dimension), the FSR

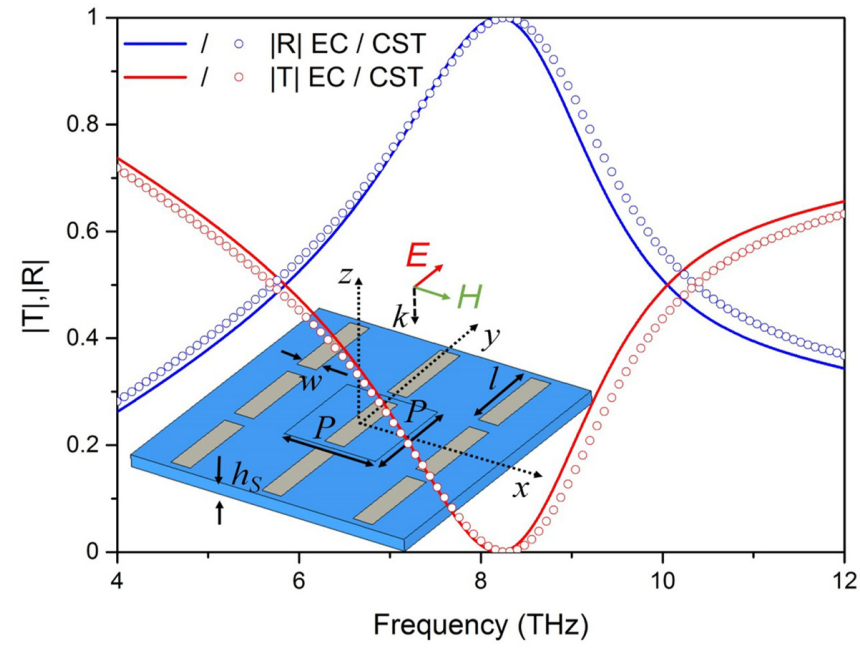

FIG. 2. EC (solid line) and full-wave simulation (circles) results for a substrate-backed patch FSR. Reflectance (blue color) and transmittance (red color). Inset: A portion of the periodic array with the geometrical parameters depicted: lattice distance, $P=14 \mu \mathrm{m}$, patch length, $l=13 \mu \mathrm{m}$, patch width, $w=2 \mu \mathrm{m}$, and substrate height, $h_{S}=5 \mu \mathrm{m} ; \varepsilon_{S}=2.25$.

is transparent within the bandwidth considered and therefore it would not be treated here.

Equivalent-circuit and full-wave simulation results for the used FSR rectangular patch array are presented in Fig. 2 to introduce the FSR structure and validate the EC approach. A portion of the whole structure along with its geometrical parameters and the chosen coordinate system are shown in the background of the figure. A normally incident wave with linear polarization along the $y$ axis, i.e., the electric field parallel to the long dimension of the patch, has been considered. As it can be observed, the agreement between the EC and fullwave simulation results is very good. In the following, we will use the EC to evaluate the performance of the absorber at selected frequency points. A time convention of the type $e^{j \omega t}$ and steady-state regime are assumed throughout this work.

Our consideration is restricted to the case when the PD layer is made of GaAs, whose complex permittivity obeys Lorentz dispersion:

$$
\varepsilon_{P D}(\omega)=\varepsilon_{\infty}+\left(\varepsilon_{0}-\varepsilon_{\infty}\right) \omega_{T}^{2} /\left(\omega_{T}^{2}-\omega^{2}+j \Gamma \omega\right),
$$

where $\varepsilon_{\infty}$ and $\varepsilon_{0}$ are the high-frequency and static permittivity, respectively, $\omega_{T}$ is the transverse phonon resonance frequency, and $\Gamma$ is the absorption factor; $\varepsilon_{\infty}=10.9, \varepsilon_{0}=$ $12.65, \omega_{T} /(2 \pi)=8.12 \times 10^{9} \mathrm{~Hz}$, and $\Gamma=0.06 \omega_{T}[33,40]$. Figure 3(a) shows the frequency dependence of GaAs permittivity, $\varepsilon_{P D}$, according to Eq. (4) [note that $\varepsilon_{P D}$ is represented as $\left.\varepsilon_{P D}=\varepsilon_{P D}^{\prime}-j \varepsilon_{P D}^{\prime \prime}\right]$. The polaritonic gap is the region where $\operatorname{Re}\left\{\varepsilon_{P D}\right\}<0$. Assuming $\Gamma=0$, the frequency limits of the polaritonic gap are set by the transverse and longitudinal phonon resonance frequencies, in line with the LyddaneSachs-Teller relation, $\omega_{L}^{2} / \omega_{T}^{2}=\varepsilon_{0} / \varepsilon_{\infty}$ [33]. These limits are slightly different in Fig. 3(a) because $\Gamma \neq 0$. In addition, the GaAs propagation constant $\gamma_{P D}$ and intrinsic impedance $Z_{P D}$ are shown in Figs. 3(b) and 3(c), respectively. These 


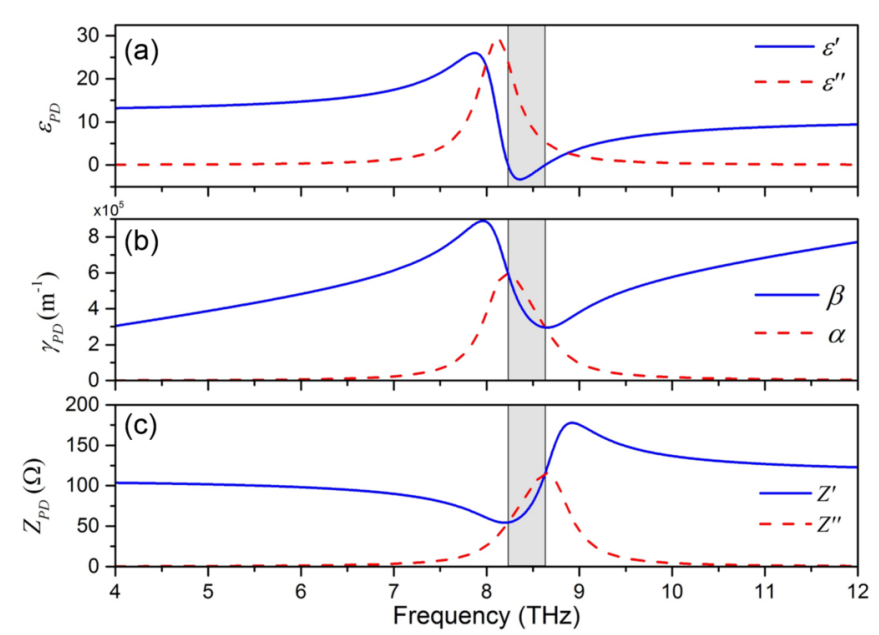

FIG. 3. (a) Relative permittivity of GaAs: real (solid blue line) and imaginary (dashed red line) parts. (b) Propagation constant inside GaAs: phase constant (solid blue line) and attenuation constant (dashed red line). (c) Intrinsic impedance of GaAs: real (solid blue line) and imaginary (dashed red line) parts. The shaded region in all panels corresponds to the polaritonic gap.

parameters are evaluated as follows:

$$
\begin{gathered}
\gamma_{P D}=\frac{j \omega}{c_{0}} \sqrt{\varepsilon_{P D}}=\alpha_{P D}+j \beta_{P D}, \\
Z_{P D}=Z_{0} \sqrt{\frac{1}{\varepsilon_{P D}}},
\end{gathered}
$$

where $\alpha_{P D}$ is the attenuation constant, $\beta_{P D}$ is the phase constant, and $c_{0}=3 \times 10^{8} \mathrm{~m} / \mathrm{s}, Z_{0} \cong 377 \Omega$ are the freespace velocity and impedance, respectively. Wherever $\varepsilon_{P D}$ has an imaginary part (also when $\operatorname{Re}\left\{\varepsilon_{P D}\right\} \approx 0$ ) the $\gamma_{P D}$ and $Z_{P D}$ are complex. This means that in order to match a PD layer of a certain thickness so that a reflectionless regime is realized, the real and imaginary parts of the impedance seen by the incident wave must be simultaneously matched to $Z_{0}$. Note that $R \rightarrow=0$ is the condition which has been commonly used in the conventional absorber concepts, i.e., without ensuring perfect one-way absorption and high transmission in the neighboring bands $[17,45,46]$. Some aspects of the use of impedance matching and EC approach for such absorbers have earlier been discussed in the literature $[23,42,46,47]$.

\section{RESULTS AND DISCUSSION}

\section{A. One-way absorption via impedance (mis)match}

To demonstrate the physical mechanism underlying oneway absorption in quasiplanar structures in Fig. 1, a systematic study was carried out by using the EC model. We selected six representative parameter sets, for which $A \rightarrow \approx 1$ is predicted by the EC model. A wide range of $\operatorname{Re}\left\{\varepsilon_{P D}\right\}$ singular regimes is covered (including $\operatorname{Re}\left\{\varepsilon_{P D}\right\} \approx 0$ and $\operatorname{Re}\left\{\varepsilon_{P D}\right\} \approx 1$ ), while the geometrical parameters are adjusted to have nearly total absorption in each case. Two of the cases, (i) and (vi), are chosen to have the maximum absorption frequency $\left(f_{\max }\right)$ outside the polaritonic gap, whereas for the rest of the cases $f_{\max }$ is located inside or very close to the polaritonic gap. They are chosen as follows: (ii) at the frequency of maximum attenuation constant $\alpha_{P D}$ (notice that it does not correspond to the frequency of maximum value of $\left|\operatorname{Im}\left\{\varepsilon_{P D}\right\}\right|$ ); (iii) and (iv) at the frequencies when $\operatorname{Re}\left\{\varepsilon_{P D}\right\} \approx 0 ;(\mathrm{v})$ at the frequency when $\operatorname{Re}\left\{\varepsilon_{P D}\right\} \approx 1$.

The value of $f_{\max }$ along with the values of $\varepsilon_{P D}, \gamma_{P D}$, and $Z_{P D}$ at that frequency and the geometrical parameters for the PD, substrate, and FSR are outlined in Table I. It is evident that the minimum PD thickness, $h_{P D}$, is obtained for case (ii) that corresponds to the maximum attenuation constant. As expected, the length of the patch, $l$, was generally reduced as the desired maximum absorption frequency is increased. The only exception is found in cases (ii) and (iii). Since their maximum absorption frequencies are very close together, the optimization process may yield a solution with the $l$ value being slightly smaller for the lower frequency case. For the rest of parameters, a direct relationship is not straightforward. However, thanks to the fine adjustment of FSR parameters with the aid of the EC model, one can achieve $A \rightarrow \approx 1$ for any frequency in the considered frequency range.

Figures 4 and 5 present the EC results for forward absorption $A^{\rightarrow}$, backward absorption $A^{\leftarrow}$, forward reflectance $R^{\rightarrow}$, backward reflectance $R^{\leftarrow}$, transmittance $T$, and forwardto-backward absorption contrast $\psi=A^{\rightarrow} / A^{\leftarrow}$, together with the corresponding full-wave simulation results. Despite small discrepancies there is very good agreement between EC and full-wave simulations within the whole frequency range. These results demonstrate that with an appropriate adjustment of the geometrical parameters of the FSR and thicknesses of PD and substrate layers, perfect absorption is attainable regardless of which operation regime in terms of $\operatorname{Re}\left\{\varepsilon_{P D}\right\}$ is realized. Clearly, to have nonzero absorption, $\operatorname{Im}\left\{\varepsilon_{P D}\right\}$ should be greater than zero, but as it will be seen later, extremely high values are not required to attain high absorption.

\begin{tabular}{|c|c|c|c|c|c|c|c|c|c|}
\hline$f_{\max }(\mathrm{THz})$ & $\varepsilon_{P D}$ & $\gamma_{P D}\left(\times 10^{5}\right)\left(\mathrm{m}^{-1}\right)$ & $Z_{P D}(\Omega)$ & $h_{P D}(\mu \mathrm{m})$ & $h_{S}(\mu \mathrm{m})$ & $h_{T}(\mu \mathrm{m})$ & $h_{T}\left(\lambda_{0}\right)^{\mathrm{a}}$ & $l(\mu \mathrm{m})$ & $w(\mu \mathrm{m})$ \\
\hline (i) 7.75 & $24.9-9 j$ & $1.5+8.2 j$ & $72+13 j$ & 0.62 & 2.21 & 2.83 & 0.073 & 13 & 3 \\
\hline (ii) 8.22 & $0.5-24.5 j$ & $5.97+6 j$ & $54+53 j$ & 0.26 & 6.33 & 6.59 & 0.180 & 12.8 & 2.94 \\
\hline (iii) 8.23 & $-0.01-23.9 j$ & $5.96+5.9 j$ & $54+54 j$ & 0.28 & 6.14 & 6.42 & 0.176 & 12.9 & 2.88 \\
\hline (iv) 8.63 & $0.01-5.3 j$ & $2.95+2.96 j$ & $115+116 j$ & 0.97 & 5.89 & 6.86 & 0.197 & 12.50 & 1.95 \\
\hline (v) 8.71 & $1-4.2 j$ & $2.3+3 j$ & $143+111 j$ & 1.34 & 5.30 & 6.64 & 0.193 & 12.43 & 2.10 \\
\hline (vi) 9.00 & $3.8-2 j$ & $0.96+3.8 j$ & $175+44 j$ & 2.74 & 1.97 & 4.71 & 0.141 & 11.96 & 2.99 \\
\hline
\end{tabular}

TABLE I. Examples of structural parameters enabling (nearly) perfect one-way absorption.

${ }^{\mathrm{a}} \lambda_{0}$ is the free-space wavelength at $f_{\max }$. 

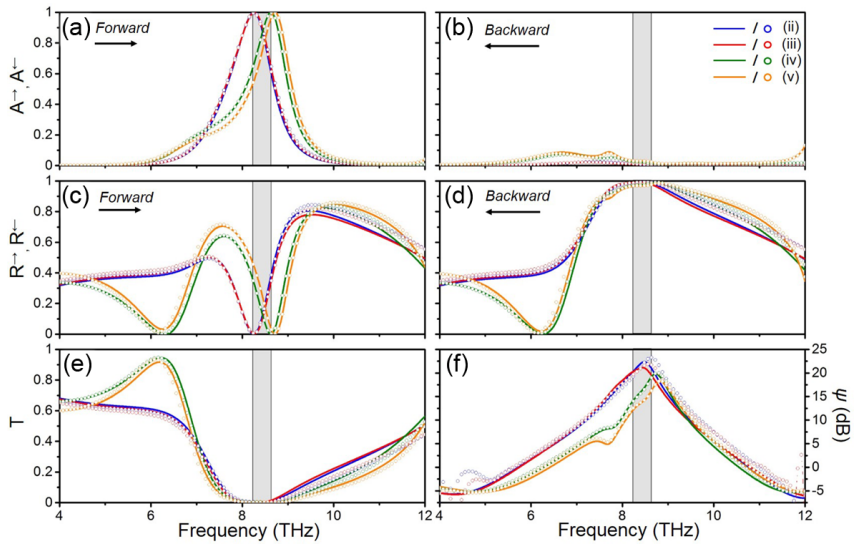

FIG. 4. EC (solid lines) and full-wave simulation (circles) results for designs (ii) blue line, (iii) red line, (iv) green line, and (v) orange line, which are outlined in Table I: (a) $A^{\rightarrow}$, (b) $A^{\leftarrow}$, (c) $R^{\rightarrow}$, (d) $R^{\leftarrow}$, (e) $T$, and (f) $\psi=A^{\rightarrow} / A^{\leftarrow}$. The shaded area depicts the location of the polaritonic gap.

The results for cases (ii)-(v) are shown in Fig. 4. They were obtained by performing a genetic algorithm optimization of the structural parameters $h_{P D}, h_{S}, l$, and $w$ (the lattice period, $P$, was kept as constant and equal to $14 \mu \mathrm{m}$ ) with the EC model, which is much faster than the numerical solver. In all cases, a maximum of $A^{\rightarrow} \approx 1$ is obtained, see Fig. 4(a).

Now, let us examine the presented designs for capability in one-way absorption. To this end, $A^{\rightarrow}, A^{\leftarrow}, R^{\rightarrow}, R^{\leftarrow}$, and $T$ are compared. As observed in Fig. 4(b), $A^{\leftarrow} \approx 0$ is obtained at the upper edge of the polaritonic gap. This feature manifests itself in the desirably large values of $\psi$, as shown in Fig. 4(f). The possibility of obtaining large values of $\psi$ gives one an idea about how to separate two illumination scenarios. To achieve large $\psi, A^{\leftarrow}$ should be minimized, and therefore $R^{\leftarrow}$ maximized. This is achieved by means of the FSR, which reflects almost perfectly in the backward illumination case at this frequency range and thus prevents any effect on the PD layer, which is the only lossy component. In turn, $R^{\rightarrow}$ is

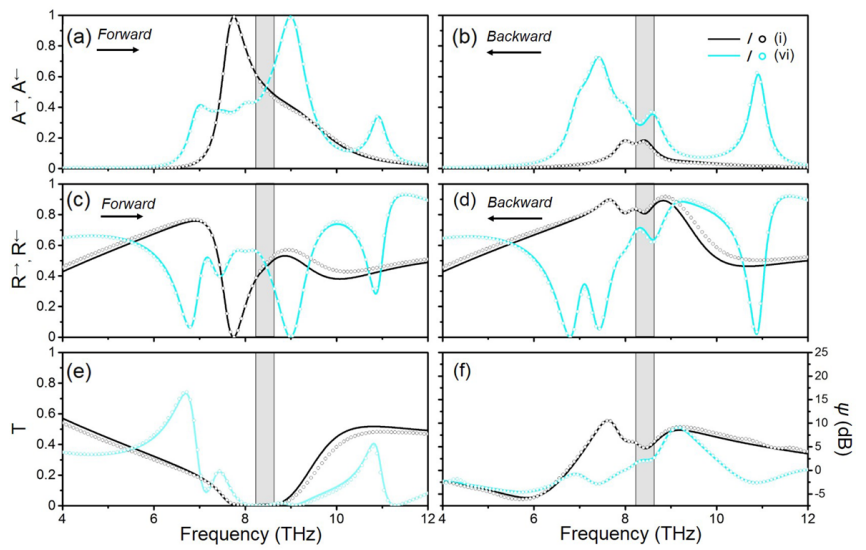

FIG. 5. EC (solid lines) and full-wave simulation (circles) results for designs (i) black line and (vi) cyan line, which are outlined in Table I: (a) $A^{\rightarrow}$, (b) $A^{\leftarrow}$, (c) $R^{\rightarrow}$, (d) $R^{\leftarrow}$, (e) $T$, and (f) $\psi=A^{\rightarrow} / A^{\leftarrow}$. The shaded area depicts the location of the polaritonic gap. minimized at the frequency of maximum absorption for all the cases. In particular, for cases (iv) and (v), an additional reflectance null at $6.2 \mathrm{THz}$ leads to a high- $T$ band, provided that the PD material has low losses at this frequency range. It should be recalled that $T$ does not depend on illumination direction, as a result of Lorentz reciprocity [41]. Summing up, among the four cases shown in Fig. 4, (ii) and (iii) offer wider absorption bands and greater $\psi$, while (iv) and (v) present an additional high- $T$ band that may confer these designs with a multifunctional property. Hence, additional optimization is required to obtain these two features in one structure.

To emphasize the importance of impedance matching, cases (i) and (vi) were studied demonstrating that $A \rightarrow \approx 1$ can be obtained even outside the polaritonic gap. Figure 5 shows the obtained EC and full-wave simulation results with excellent agreement. For case (i), the maximum of $A^{\rightarrow} \approx 1$ is obtained at approximately $7.75 \mathrm{THz}$ [Fig. 5(a)]. However, $A^{\leftarrow}$ remains significant at this frequency, leading to a less competitive solution in terms of $\psi$ as compared to cases (ii)-(v). Moreover, $T$ is not high at the neighboring bands. It should be noted that $h_{P D}$ is only $0.62 \mu \mathrm{m}$ in case (i) [the minimum $h_{P D}=0.26 \mu \mathrm{m}$ is achieved in case (ii)]. Case (i) is the most compact structure with a total thickness of $2.83 \mu \mathrm{m}$, i.e., just $0.073 \lambda_{0}$.

For case (vi), the maximum $A \rightarrow$ is obtained near $9 \mathrm{THz}$, see Fig. 5(a). In this case, absorption bands with a moderately high $A^{\leftarrow}$ appear at 7.5 and $11 \mathrm{THz}$, as shown in Fig. 5(b). At these frequencies, the transmittance through the FSR patch can be kept relatively high. Similarly to case (i), $\psi$ is relatively low compared to cases (ii)-(v). A peak of $T=0.75$ at $6.7 \mathrm{THz}$ is noticeable.

The relation between the one-way absorption maxima and structural parameters can be easily understood in terms of impedance matching. Figures 6 and 7 show $Z_{\text {in }} \overrightarrow{\text { and }} Z_{\text {in }}^{\leftarrow}$ versus $f$, obtained from the EC model. To get perfect impedance matching at the air-PD interface and thus fulfill the condition of $R^{\rightarrow}=0$, it is required that

$$
\operatorname{Re}\left\{Z_{\text {in }}^{\vec{n}}\right\}=Z_{0}, \quad \operatorname{Im}\left\{Z_{\text {in }}^{\overrightarrow{1}}\right\}=0 .
$$
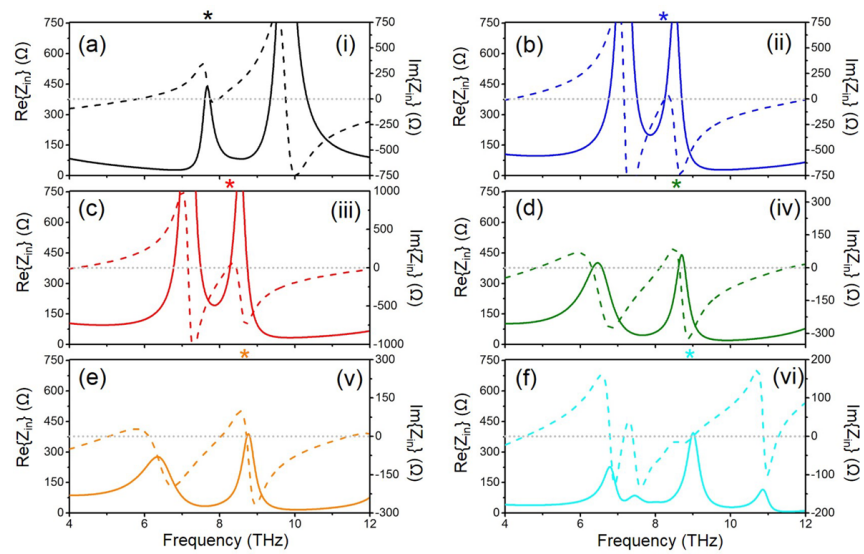

FIG. 6. Input impedance for forward illumination $\left(Z_{\text {in }}\right)$. Real (solid lines) and imaginary (dashed lines) parts are depicted: (a) case (i), (b) case (ii), (c) case (iii), (d) case (iv), (e) case (v), and (f) case (vi). The dash-dotted horizontal line corresponds to $\operatorname{Re}\left\{Z_{\text {in }}\right\}=\eta_{0}$ and $\operatorname{Im}\left\{Z_{\text {in }}\right\}=0$. The symbol $*$ denotes the maximum absorption frequency for each case. 

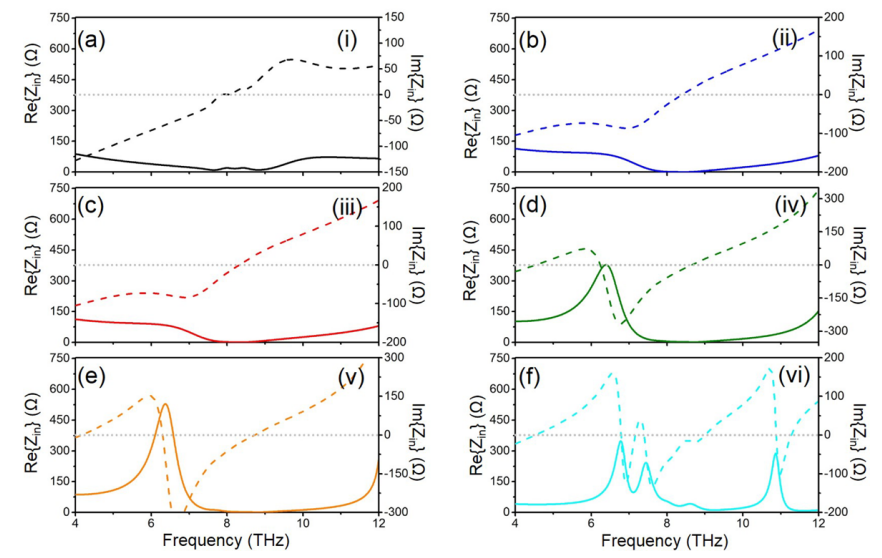

FIG. 7. Input impedance $\left(Z_{\text {in }}^{\leftarrow}\right)$ for backward illumination. Real (solid lines) and imaginary (dashed lines) parts are depicted: (a) case (i), (b) case (ii), (c) case (iii), (d) case (iv), (e) case (v), and (f) case (vi). The dash-dotted horizontal line corresponds to $\operatorname{Re}\left\{Z_{\text {in }}\right\}=\eta_{0}$ and $\operatorname{Im}\left\{Z_{\text {in }}\right\}=0$.

If Eq. (7) is satisfied, the wave incident on the air-PD interface in the forward illumination case is expected to be transmitted without reflection to the PD layer, so perfect absorption may be achieved. It is worth noting that Eq. (7) is equivalent to $R \rightarrow=$ 0 , so it represents a necessary but not sufficient condition of perfect absorption. In fact, whether high absorption or hightransmission regimes are realized in this case depends on the other conditions.

As observed in Fig. 6, the conditions (7) are approximately satisfied at the maximum of $A \rightarrow$ for all the considered cases, i.e., from (i) to (vi). Hence, the key role of impedance matching is evident. Moreover, particular absorption features shown in Figs. 4 and 5 can be explained in terms of input impedance. For instance, in cases (ii) and (iii) a broader absorption response was observed around the maximum $A \rightarrow$. This performance is connected with a smoother transition of $\operatorname{Im}\left\{Z_{\text {in }}\right\}$ through zero than in the remaining cases, see Fig. 6(d) and 6(e).

Although the variation of $\operatorname{Re}\left\{Z_{\text {in }}\right\}$ is relatively abrupt, the influence of $\operatorname{Im}\left\{Z_{\text {in }}^{\rightarrow}\right\}$ has a major impact on the matching. As expected, impedance matching also plays a key role for the high- $T$ band appearing in cases (iv), (v), and (vi). For case (iv), where $T$ is maximum, both conditions in Eq. (7) are fulfilled, see Fig. 6(d). In combination with low attenuation within the PD layer, this leads to almost total transmission, as observed in Fig. 4(e). For cases (v) and (vi), only the condition for $\operatorname{Im}\left\{Z_{\text {in }}\right\}$ is attained [Figs. 6(e) and 6(f)] and nevertheless, relatively high $T$ is predicted for case (v) [Fig. 4(e)].

Impedance mismatch at backward illumination is required to block transmission, which is the same for both illumination directions due to the reciprocity. Hence, if $T=0$, Eq. (7) predicts high $A^{\rightarrow}$ and high $R^{\leftarrow}$, as demonstrated above. Input impedance under backward illumination $Z_{\text {in }}^{\leftarrow}$ is presented in Fig. 7. It is seen that at the rejection band the impedance tends to a short circuit, namely, $Z_{\text {in }}^{\leftarrow} \approx 0$. For cases (i), (ii), and (iii), impedance matching is not fulfilled at any frequency and therefore $A^{\leftarrow}=1$ and/or high $T$ were not attainable. However, for cases (iv), (v), and (vi), there are frequencies wherein a good impedance matching is observed. Then, high $T$ can be achieved when attenuation in the PD layer is low, see Fig. 4(e).
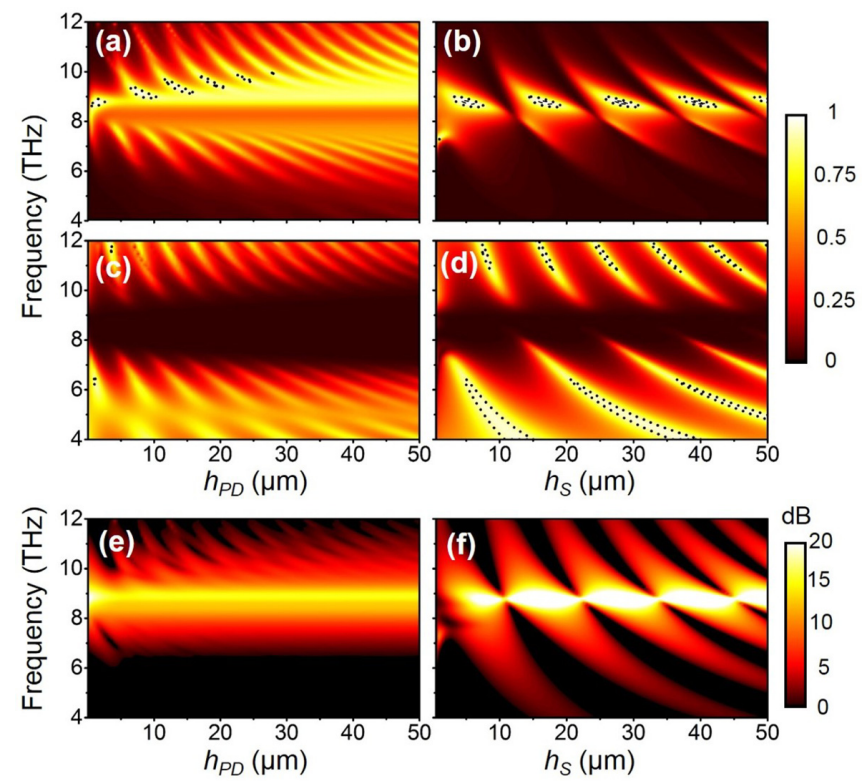

FIG. 8. (a, b) $A^{\rightarrow}$, (c, d) $T$, and (e, f) $\psi$ at varying $h_{P D}$ and $h_{S}$ : (a, c, e) $h_{P D}$ is varied and $h_{S}=5.3 \mu \mathrm{m}$; (b, d, f) $h_{S}$ is varied and $h_{P D}=1.34 \mu \mathrm{m}$. Dashed isolines at panels (a), (b) and (c), (d) correspond to $A^{\rightarrow}=0.9$ and $T=0.9$, respectively.

In turn, if attenuation is significant, some absorption can be experienced, as demonstrated for the $A \leftarrow$ peaks appearing for case (vi) at 7.5 and $11 \mathrm{THz}$.

To summarize, the case of $A^{\rightarrow} \approx 1$ can be obtained by forward impedance matching, which depends on both $\operatorname{Re}\left\{Z_{\mathrm{in}}^{\rightarrow}\right\}$ and $\operatorname{Im}\left\{Z_{\text {in }}\right\}$. It can be retrieved from the EC model results in a wide range of $\varepsilon_{P D}$ variation. High attenuation in the PD layer is itself not crucial for high absorption but it is still an important part of the resulting matching mechanism. However, for the simultaneous fulfillment of $A^{\rightarrow}=1$ and $A^{\leftarrow}=0$, only the range in which the FSR provides $R^{\leftarrow}=1$ and high contrast is useful, so cases (ii)-(v) are preferable.

\section{B. Different absorption regimes by varying geometry}

In Sec. III A, several selected designs have been analyzed in the context of one-way absorption. In this section, design (v) is taken as an entry point for a parametric study of the effects of $h_{P D}$ and $h_{S}$ with the aim to demonstrate their contribution to the different absorption regimes. Note that $\operatorname{Re}\left\{\varepsilon_{P D}\right\}$ varies quickly at the polaritonic gap range and takes each of the zero and unity values twice, i.e., near $f=\omega_{T} /(2 \pi)$ and $f=\omega_{L} /(2 \pi)$, so possible contributions of ENZ and the transparency regimes might not be well distinguishable. For instance, $\operatorname{Re}\left\{\varepsilon_{P D}\right\}=1$ at $f=8.71 \mathrm{THz}$, while $\operatorname{Re}\left\{\varepsilon_{P D}\right\} \approx 0$ at $f=8.23 \mathrm{THz}$ and $8.63 \mathrm{THz}$, see Table I. The abovementioned quick variation provides the possibility of the required (mis)match within a narrow frequency range.

Figure 8 shows the $\left(h_{P D}, f\right)$-plane and $\left(h_{S}, f\right)$-plane maps of $A^{\rightarrow}, T$, and $\psi$, obtained with the EC approach. First, $h_{P D}$ was varied from 0.05 to $50 \mu \mathrm{m}$ with a step of $0.05 \mu \mathrm{m}$ by keeping $h_{S}=5.3 \mu \mathrm{m}$ [see Figs. 8(a), 8(c) and 8(e)]. Then $h_{S}$ was varied from 1 to $50 \mu \mathrm{m}$ with a step of $0.5 \mu \mathrm{m}$ by 
keeping $h_{P D}=1.34 \mu \mathrm{m}$ [see Figs. 8(b), 8(d) and 8(f)]. We first consider the effects exerted by variations in $h_{P D}$.

At least three regimes can be distinguished in Fig. 8(a), depending on the behavior of $A \rightarrow$. Dashed black lines are plotted as isolines for $A^{\rightarrow}=0.9$ in order to highlight that the regions of maximum absorption are achieved owing to appropriate impedance matching. These regions can be particularly important for one-way absorption.

In the first regime, which corresponds to the vicinity of $f=$ $\omega_{L} /(2 \pi)=8.75 \mathrm{THz}$, high absorption occurs for any value of $h_{P D}$. This is due to the coexistence of high attenuation within the PD layer and good impedance matching, which is preserved for the entire range of $\varepsilon_{P D}$ variation.

In the second regime, which corresponds to the frequencies above and below the polaritonic gap, high absorption is connected with Fabry-Perot resonances. They lead to the observed fringes of $A \rightarrow$ and $T$. The inclination and separation of individual fringes from their neighbors depend on $h_{P D}$ and $\varepsilon_{P D}$. This behavior is realized in Fig. 8 for a broad frequency band and in a wide range of $h_{P D}$, starting from $4 \mu \mathrm{m}$.

The third regime can be assigned rather to the vicinity of $f=\omega_{T} /(2 \pi)=8.12 \mathrm{THz}$, where strong absorption can appear due to impedance matching at small values of $h_{P D}$ (see also Fig. 10). In this case, $h_{P D}$ must be chosen as a tradeoff between the attenuation distance required for total absorption and the impedance matching condition. Indeed, the former condition requires sufficiently thick PD layers, while the latter is more easy to attain for thin PD layers. It should be noticed that for thick PD layers the impinging wave is strongly attenuated and then it is influenced to a lesser extent by the subsequent layers, namely, by the substrate and FSR. In contrast, for thin PD layers they can contribute to the impedance matching.

It has been found that not all the parts of the high- $A^{\rightarrow}$ region in Fig. 8(a) yield one-way absorption. Indeed, since a FSR enables $T \approx 0$ in a narrow frequency range, large values of $\psi$ are expected to be achievable only in this range, as it is observed in Fig. 8(e). Thus, a possible contribution of the first and third regimes to one-way absorption is more important. In Fig. 8(c), one can see that wide fringes of high transmission appear at $f<7 \mathrm{THz}$, while small values of $h_{P D}$ are especially suitable for obtaining larger regions of high transmission in the $\left(h_{P D}, f\right)$ plane. Once high-efficiency transmission bands adjacent to the absorption band are obtained, the desired multifunctional operation can in principle be realized.

Variations in $h_{S}$ give the second degree of freedom for oneway absorbers. In contrast with the variations in $h_{P D}$, strong absorption happens only for some ranges of $h_{S}$ variation. An example is presented in Figs. 8(b), 8(d) and 8(f) by fixing $h_{P D}=1.34 \mu \mathrm{m}$. The modulation of $A^{\rightarrow}$ and $T$ at varying $h_{S}$ takes place, so $A^{\rightarrow}$ can be maximized. This gives evidence of the fact that the effect of the absorbing layer strongly depends on the lossless substrate parameters as a consequence of impedance (mis)match. Hence, the same portion of the absorbing material can yield either strong or weak absorption, see Fig. 8(b). Simultaneously, wide ranges of high $T$ appear when $f<8 \mathrm{THz}$. If a frequency cut is taken in Fig. 8(b), say, at $f=8.5 \mathrm{THz}\left(\lambda_{S}=23.5 \mu \mathrm{m}\right)$, the regions of high absorption are periodically spaced by $0.5 \lambda_{S}$. This indicates that the high absorption is obtained due to Fabry-Perot resonances in the

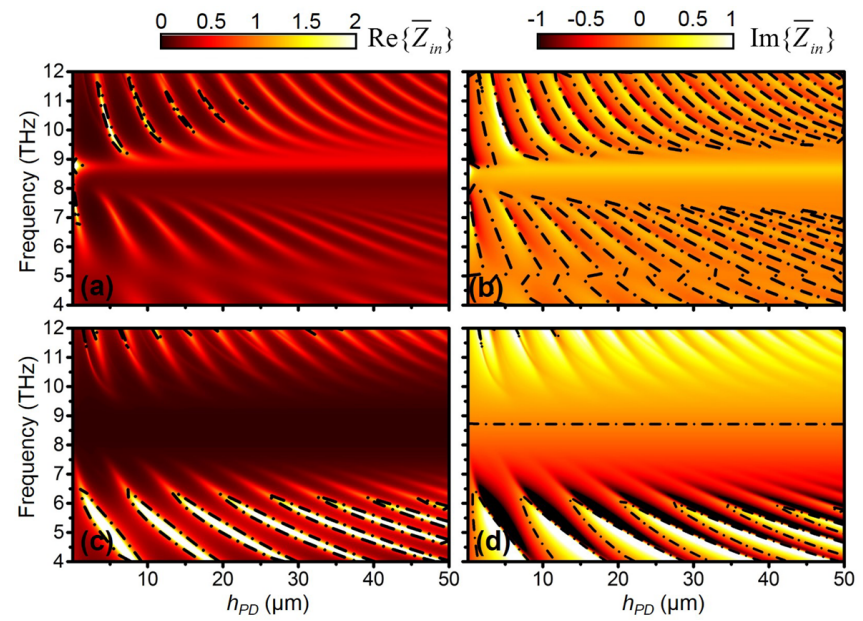

FIG. 9. Normalized input impedance, $\bar{Z}_{\text {in }}:$ (a) $\operatorname{Re}\left\{\bar{Z}_{\text {in }}\right\}$, (b) $\operatorname{Im}\left\{\bar{Z}_{\text {in }}\right\},\left(\right.$ c) $\operatorname{Re}\left\{\bar{Z}_{\text {in }}^{\leftarrow}\right\}$, and (d) $\operatorname{Im}\left\{\bar{Z}_{\text {in }}^{\leftarrow}\right\}$.

substrate layer. Due to universality of the EC model, these resonances can be taken into account in terms of $Z_{\text {in }}$. Also $T$ depends strongly on $h_{S}$, see Fig. 8(d). For instance, the range $5<h_{S}<6.5 \mu \mathrm{m}$ is promising for coexistence of absorption and neighboring transmission bands. From the results presented in Fig. 8, the generality of the suggested approach to one-way absorption is clearly seen. Indeed, high $\psi$ can be obtained in wide ranges of variation in $h_{P D}$ and $h_{S}$, even though the regions where the forward-case impedance matching is better and thus the highest $A^{\rightarrow}$ values achieved may appear outside the region of highest $\psi$. The only exception is related to very small values of $h_{P D}$, e.g., less than $2 \mu \mathrm{m}$. Hence, the use of very thin absorbing layers can be preferable also because of possible coincidence of the regions of high $A^{\rightarrow}$ and high $\psi$.

For further evidence, Fig. 9 shows the normalized input impedance $\bar{Z}_{\text {in }}=Z_{\text {in }} / Z_{0}$ under forward illumination $Z_{\text {in }}$ and under backward illumination $Z_{\text {in }}^{\leftarrow}$ for the whole range of $h_{P D}$ variation. The dash-dotted black lines in Figs. 9(a) and 9(c) and Figs. 9(b) and 9(d) correspond to $\operatorname{Re}\left\{\overline{Z_{\text {in }}}\right\}=1$ and $\operatorname{Im}\left\{\overline{Z_{\text {in }}}\right\}=0$, respectively. To obtain perfect absorption in the forward case, both conditions in Eq. (7) should simultaneously be fulfilled. It is clear that for relatively large values of $h_{P D}$ the input impedance does not vary anymore within the polaritonic gap region. This happens due to the fact that the incident wave is partially reflected at the air-PD interface and partially attenuated in the PD layer. Due to high attenuation, the incident wave cannot reach the substrate and FSR layers, and hence they have no impact on $Z_{\text {in. }}$. This is the reason why regions of a relatively weak sensitivity to $h_{P D}$ variations are observed for $A^{\rightarrow}$ and $\psi$ in Figs. 8(a) and 8(e), respectively. Consequently, for large values of $h_{P D}$, the case of $A^{\rightarrow}=1$ is not attainable inside and around the polaritonic gap. Regarding backward illumination results, it can be observed that $Z_{\text {in }}^{\leftarrow}$ is close to zero for the rejection band provided by the FSR element. Between 4 and $6 \mathrm{THz}$ there are some regions wherein impedance matching can be fulfilled. However, due to the low attenuation inside the PD layer at those frequencies high $A^{\leftarrow}$ is not possible.

According to the obtained results, high forward absorption $A^{\rightarrow}$ occurs even when $h_{P D}<1 \mu \mathrm{m}$, so ultrathin PD layers still enable high absorption. This mechanism is illustrated in 


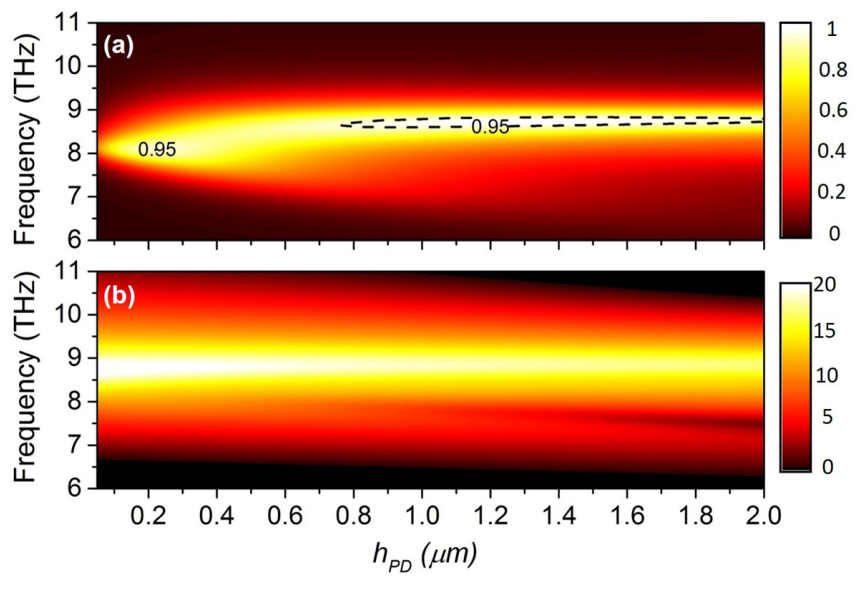

FIG. 10. Zoomed view of Figs. 8(a) and 8(e) at small values of $h_{P D}:$ (a) $A^{\rightarrow}$, (b) $\psi$.

Fig. 10, where detailed views of Figs. 8(a) and 8(e) in the interval $0.05<h_{P D}<2 \mu \mathrm{m}$ are displayed. In this case, the highest $A^{\rightarrow}$ appears simultaneously with the highest $\psi$. There are two regions with $A^{\rightarrow}>0.95$ that appear at $f \approx 8.2 \mathrm{THz}$ for $h_{P D} \approx 0.25 \mu \mathrm{m}$ (very small region, third regime), and at $f \approx 8.7 \mathrm{THz}$ for $0.8<h_{P D}<1.9 \mu \mathrm{m}$ (large region, first regime). These regions correspond to the results obtained for the patch FSR designs in Sec. III A.

Finally, a zoomed view of a fragment of Fig. 9 is presented in Fig. 11 for the same $h_{P D}$ range as in Fig. 10. It can be observed that at the region of maximum absorption (near-)matching conditions are fulfilled. The contours where matching occurs are shown by dash-dotted lines representing $\operatorname{Re}\left\{\overline{Z_{\text {in }}}\right\}=1$ and $\operatorname{Im}\left\{\overline{Z_{\text {in }}}\right\}=0$ in Figs. 11(a) and 11(b), respectively. In turn, mismatching is observed for backward illumination for a wide frequency range [see Figs. 11(c) and 11(d)]. What is more, due to the selection of the FSR geometrical parameters a short-circuit condition, i.e., $\operatorname{Re}\left\{\overline{Z_{\text {in }}}\right\}=0$ and $\operatorname{Im}\left\{\overline{Z_{\text {in }}}\right\}=0$, and therefore total reflection is expected to occur.

Generally, comparison of the results in Figs. 8 and 10 with those in Figs. 9 and 11 confirms the expectation that matching
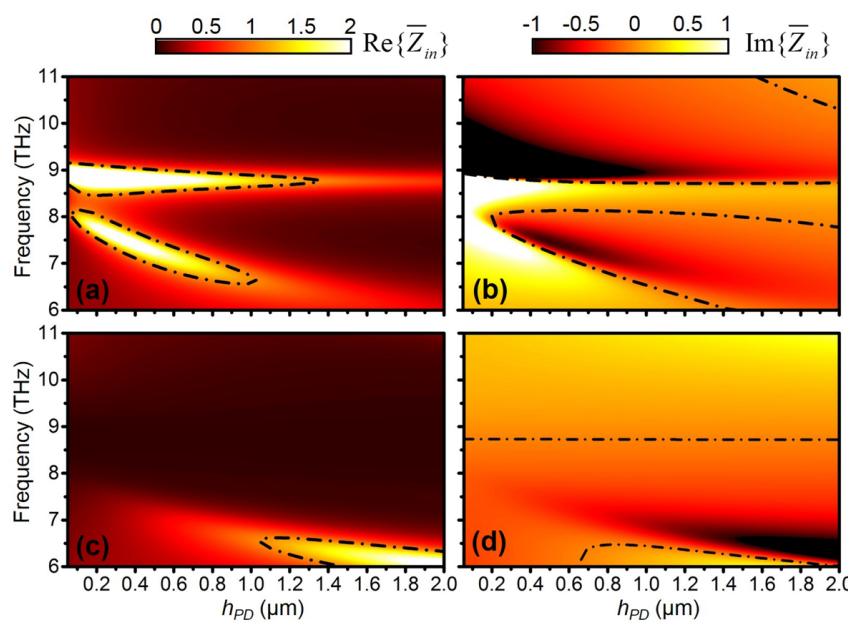

FIG. 11. Zoomed view of Fig. 9 at small values of $h_{P D}$ : (a) $\operatorname{Re}\left\{\bar{Z}_{\text {in }}\right\},\left(\right.$ b) $\operatorname{Im}\left\{\bar{Z}_{\text {in }}\right\}$, (c) $\operatorname{Re}\left\{\bar{Z}_{\text {in }}^{\leftarrow}\right\}$, and (d) $\operatorname{Im}\left\{\bar{Z}_{\text {in }}^{\leftarrow}\right\}$. conditions are more important for obtaining a high absorption band than the specific values of $\operatorname{Re}\left\{\varepsilon_{P D}\right\}$ and $\operatorname{Im}\left\{\varepsilon_{P D}\right\}$, which is in principal coincidence with recent theories and results [23].

\section{CONCLUSION}

To summarize, a general concept of quasiplanar one-way $\mathrm{THz}$ absorbers based on polar dielectrics, which combine (nearly) perfect impedance matching in forward illumination and impedance mismatch in backward illumination, has been proposed and validated. A thorough demonstration is carried out for the case when transparency bands may appear in a close neighborhood of one-way absorption bands, and they all can be explained in the framework of the unified impedance model. Although this performance can be achieved at rather arbitrary $\varepsilon_{P D}$, the fine tuning of both real and imaginary parts of the PD permittivity remains critical. Generally, structures with broken spatial inversion symmetry that enable realization of this concept comprise homogeneous absorbing and substrate layers and a FSR. They have simple geometry and require conventional materials like GaAs and polypropylene. The study has been carried out by using an analytical EC approach and full-wave simulations. The results are in excellent agreement, so the former can provide a valuable physical insight to understand the different operation regimes. The conditions necessary for perfect one-way absorption that originate from the energy conservation and Lorentz reciprocity are equivalent to the criteria of impedance matching at forward illumination and mismatching at backward illumination, which are used within the EC approach. At the backward-case mismatch, transmission is vanishing for both illumination directions, so the ambiguity of the forward-case matching conditions is resolved, and all of them combined can properly predict oneway absorption regimes. The role of choice of the thicknesses of individual (absorbing and substrate) layers in realization of one-way absorbers has been analyzed. It has been shown that high forward-to-backward absorption contrast can be obtained in a very wide range of thickness of the absorbing layer, although small thicknesses are preferable to overlap the regions of high forward absorption and high contrast. High-efficiency transmission bands can appear around the absorption band, as desired for simultaneous absorption- and transmission-related regimes at close frequencies. Hence, such coexistence of the bands is promising from a multifunctionality perspective. The obtained results may serve a suitable starting point for future studies of particular practical cases. For instance, this kind of device could be used in combination with $\mathrm{THz}$ sources to obtain single-frequency signals, in multifrequency reflector systems and for passive cooling applications. In addition, they illustrate the generality of the suggested concept and universality of the EC model and impedance matching interpretation.

Perfect one-way absorption has been demonstrated for an extremely compact structure with a total thickness about $0.073 \lambda_{0}$. There is no restriction to the FSR type, i.e., other FSR geometries besides patches can be used, including those based on metasurfaces. While only GaAs has been considered in this paper, the presented approach based on the EC model and impedance (mis)match can be extended towards rather arbitrary combinations of material and geometrical parameters 
of the absorbing layer. For instance, PDs other than GaAs, such as $\mathrm{LiF}, \mathrm{NaCl}$, and $\mathrm{SiC}$, can be utilized.

\section{ACKNOWLEDGMENTS}

This work is supported by the Spanish Ministerio de Economía y Competitividad (MINECO) (TEC2014-51902-
C2-2-R). P.R.U. is sponsored by the Public University of Navarra via a predoctoral scholarship, and A.E.S. is supported by the National Science Center of Poland (NCN) (MetaSel DEC-2015/17/B/ST3/00118). We are very thankful to Prof. S. Nojima, YCU (Yokohama, Japan), for valuable discussions.
[1] M. Tonouchi, Nat. Photonics 1, 97 (2007).

[2] G. T. Ruck, D. E. Barrick, W. D. Stuart, and C. K. Krichbaum, Radar Cross Section Handbook (Plenum Press, New York, 1970), Vol. 1 .

[3] M.-J. Park, J. Choi, and S.-S. Kim, IEEE Trans. Magn. 36, 3272 (2000).

[4] Y. Avitzour, Y. A. Urzhumov, and G. Shvets, Phys. Rev. B 79, 045131 (2009).

[5] X. Liu, T. Starr, A. F. Starr, and W. J. Padilla, Phys. Rev. Lett. 104, 207403 (2010)

[6] N. I. Landy, S. Sajuyigbe, J. J. Mock, D. R. Smith, and W. J. Padilla, Phys. Rev. Lett. 100, 207402 (2008).

[7] K. B. Alici, A. B. Turhan, C. M. Soukoulis, and E. Ozbay, Opt. Express 19, 14260 (2011).

[8] K. Aydin, V. E. Ferry, R. M. Briggs, and H. A. Atwater, Nat. Commun. 2, 517 (2011).

[9] Q. Feng, M. Pu, C. Hu, and X. Luo, Opt. Lett. 37, 2133 (2012).

[10] M. A. Kats, R. Blanchard, P. Genevet, and F. Capasso, Nat. Mater. 12, 20 (2013).

[11] W. Wu and R. Magnusson, Opt. Lett. 37, 2103 (2012).

[12] Y. Ra'di, V. S. Asadchy, and S. A. Tretyakov, IEEE Trans. Antennas Propag. 61, 4606 (2013).

[13] Y. D. Chong, L. Ge, H. Cao, and A. D. Stone, Phys. Rev. Lett. 105, 053901 (2010).

[14] Y. Jin, S. Xiao, N. A. Mortensen, and S. He, Opt. Express 19, 11114 (2011).

[15] S. Feng and K. Halterman, Phys. Rev. B 86, 165103 (2012).

[16] S. Campione, I. Brener, and F. Marquier, Phys. Rev. B 91, 121408 (2015).

[17] B. C. Sturmberg, T. K. Chong, D.-Y. Choi, T. P. White, L. C. Botten, K. B. Dossou, C. G. Poulton, K. R. Catchpole, R. C. McPhedran, and C. M. de Sterke, Optica 3, 556 (2016).

[18] A. E. Serebryannikov, S. Nojima, K. Alici, and E. Ozbay, J. Appl. Phys. 118, 133101 (2015).

[19] J. Yoon, M. Zhou, M. A. Badsha, T. Y. Kim, Y. C. Jun, and C. K. Hwangbo, Sci. Rep. 5, 12788 (2015).

[20] T. S. Luk, S. Campione, I. Kim, S. Feng, Y. C. Jun, S. Liu, J. B. Wright, I. Brener, P. B. Catrysse, S. Fan et al., Phys. Rev. B 90, 085411 (2014).

[21] A. E. Serebryannikov, S. Nojima, and E. Ozbay, Phys. Rev. B 90, 235126 (2014).

[22] C. M. Watts, X. Liu, and W. J. Padilla, Adv. Mater. 24, OP98 (2012).

[23] Y. Ra'di, C. R. Simovski, and S. A. Tretyakov, Phys. Rev. Appl. 3, 037001 (2015).
[24] M. Veysi, C. Guclu, O. Boyraz, and F. Capolino, J. Opt. Soc. Am. B 32, 318 (2015).

[25] E. Colak, A. E. Serebryannikov, A. Ozgur Cakmak, and E. Ozbay, Appl. Phys. Lett. 102, 151105 (2013).

[26] M. Mutlu, S. Cakmakyapan, A. E. Serebryannikov, and E. Ozbay, Phys. Rev. B 87, 205123 (2013).

[27] T. Jang, H. Youn, Y. J. Shin, and L. J. Guo, ACS Photonics 1, 279 (2014)

[28] J. Shi, M. E. Pollard, C. A. Angeles, R. Chen, J. C. Gates, and M. D. B. Charlton, Sci. Rep. 7, 1812 (2017).

[29] H. Ramezani, Y. Wang, E. Yablonovitch, and X. Zhang, IEEE J. Sel. Top. Quantum Electron. 22, 115 (2016).

[30] R. Rodríguez-Berral, F. Mesa, and F. Medina, IEEE Trans. Antennas Propag. 63, 1969 (2015).

[31] CST Microwave Studio (2017) (accessed June 2017), http://www.cst.com.

[32] E. Rephaeli, A. Raman, and S. Fan, Nano Lett. 13, 1457 (2013).

[33] C. Kittel, Introduction to Solid State Physics (John Wiley and Sons, New York, 2005).

[34] A. E. Serebryannikov, E. Ozbay, and S. Nojima, Opt. Express 22, 3075 (2014).

[35] M. M. Sigalas, C. M. Soukoulis, C. T. Chan, and K. M. Ho, Phys. Rev. B 49, 11080 (1994).

[36] S. Nojima, Phys. Rev. B 57, R2057 (1998).

[37] S. Foteinopoulou, M. Kafesaki, E. N. Economou, and C. Soukoulis, Phys. Rev. B 84, 035128 (2011).

[38] M. Kafesaki, A. Basharin, E. Economou, and C. Soukoulis, Photonics Nanostruct. Fundam. Appl. 12, 376 (2014).

[39] M. Massaouti, A. Basharin, M. Kafesaki, M. Acosta, R. Merino, V. Orera, E. Economou, C. Soukoulis, and S. Tzortzakis, Opt. Lett. 38, 1140 (2013).

[40] R. I. Merino, M. F. Acosta, and V. M. Orera, J. Eur. Ceram. Soc. 34, 2061 (2014).

[41] R. J. Potton, Rep. Prog. Phys. 67, 717 (2004).

[42] H.-T. Chen, Opt. Express 20, 7165 (2012).

[43] R. Ortuño, C. García-Meca, and A. Martínez, Plasmonics 9, 1143 (2014).

[44] D. M. Pozar, Microwave Engineering (John Wiley \& Sons, New York, 2009).

[45] Y. Yao, R. Shankar, M. A. Kats, J. Kong, M. Loncar, and F. Capasso, Nano Lett. 14, 6526 (2014).

[46] M. Pu, P. Chen, Y. Wang, Z. Zhao, C. Wang, C. Huang, C. Hu, and X. Luo, Opt. Express 21, 11618 (2013).

[47] M. P. Hokmabadi, D. S. Wilbert, P. Kung, and S. M. Kim, Opt. Express 21, 16455 (2013). 